\section{Remarks on Extinguishment and the Response Function for a Burning Solid Propellant}

\author{
F. E. C. Culick* \\ California Institute of Technology, Pasadena, Calif.
}

$\mathbf{T}$ $\mathrm{HE}$ presence of small amplitude pressure oscillations in a solid rocket chamber depends mainly upon the interaction between the vibrations and the combustion processes occurring in the thin region adjacent to the solid. Often the behavior seems to be dominated by the influence of pressure fluctuations; this is conveniently expressed in terms of the response function $\left(\mathrm{m}^{\prime} / \bar{m}\right) /\left(p^{\prime} / \bar{p}\right)$, the ratio of a small change in mass flux to a small change of pressure. Most calculations lead to results that can be put in the form ${ }^{1}$ for harmonic oscillations

$\left(m^{\prime} / \bar{m}\right) /\left(p^{\prime} / \bar{p}\right)=n A B /[\lambda+A / \lambda-(1+A)+A B]$

where $\lambda$ is a complex function of the dimensionless frequency, $\Omega=\varkappa \omega / \bar{r}^{2}$. The angular frequency is $\omega, \bar{r}$ is the mean burning rate, $x$ is the thermal diffusivity of the solid, and the pressure index is $n$ appearing in the burning rate law $\bar{r} \sim \bar{p}^{n}$.

There are two parameters in this simple result: 1) $A=$ $E_{s}\left(\bar{T}_{s}-T_{0}\right) / R_{0} \bar{T}_{s}^{2}$, where $E_{s}$ is the activation energy for the surface reaction, $\bar{T}_{s}$ is the mean surface temperature, and $T_{0}$ is the temperature of the cold solid; and 2 ) $B$, which depends on the model chosen for the gas-phase flame. A currently pressing problem is to identify the values of $A$ and $B$ for particular propellants. One way to do this experimentally may be to use data taken in $T$ and $L^{*}$ burners. $^{2}$ If successful, one would then have a means of characterizing the stability properties of solid propellants. A possible alternate method is suggested here.

Now Eq. (1) is really just the frequency response for a special linear system. It can therefore be used to compute the response of the burning solid to any kind of pressure change. One must assume, of course, that the structure of the flame zone in some sense does not change. It appears that the most important assumption in this respect is that the gas phase responds quasi-statically to the pressure change. ${ }^{1,2}$

In particular, an approximately exponential pressure change is relatively easy to reproduce experimentally by opening or closing a vent in a small chamber. The case of decreasing pressure is important for the practical problem of extinguishment; a suitable representation is

$$
p^{\prime} / \bar{p}=\Delta\left(1-e^{-\beta \tau}\right)
$$

with $\Delta<0$ and $\tau$ is the dimensionless time, $t \bar{r}^{2} / x$. Providing the pressure changes are not too large, the response of a burning solid to any pressure change can be found by treating (1)

Received February 6, 1969; revision received March 19, 1969. This work was partially supported by the Jet Propulsion Laboratory, California Institute of Technology.

* Associate Professor, Daniel and Florence Guggenheim Jet Propulsion Center, Kármán Laboratory of Fluid Mechanies and Jet Propulsion. Member AIAA. as a Laplace transform. It appears that the first calculation of this sort appeared in Ref. 3, but not for an exponential change and with no intention of applying the results to experimental data. For the function (2), one eventually finds in this way

$$
\begin{gathered}
\frac{\exp \left(-\tau^{\prime}\right)}{2 n A B \Delta} \frac{m^{\prime}}{\bar{m}}=\sum_{i=1}^{2}\left(E_{i}\right) \sigma_{i} \exp \left(\sigma_{i}^{2} \tau^{\prime}\right) \operatorname{erfc}\left[-\sigma_{i}\left(\tau^{\prime}\right)^{1 / 2}\right]+ \\
D_{3} \exp \left(\tau^{\prime}\right) \operatorname{erfc}\left(-\tau^{\prime}\right)^{1 / 2}-\sigma_{3} \exp \left(\sigma_{3}^{2} \tau^{\prime}\right) \times \\
\left\{C_{3} \operatorname{erfc}\left[-\sigma_{3}\left(\tau^{\prime}\right)^{1 / 2}\right]-C_{4} \operatorname{erfc}\left[\sigma_{3}\left(\tau^{\prime}\right)^{1 / 2}\right]\right\} \\
\text { where } \tau^{\prime}=\tau / 4, \sigma_{3}{ }^{2}=1-4 \beta, \sigma_{2}=\sigma_{1}^{*}, \text { and } \\
\sigma_{1}=A(1-B)+i[4 A-(A-A B+1)]^{1 / 2}
\end{gathered}
$$

The coefficients are

$$
\begin{aligned}
E_{1}=C_{1}-D_{1}= & \frac{\left(1+\sigma_{1}\right)}{\left(\sigma_{1}-\sigma_{1}^{*}\right)\left(\sigma_{1}^{2}-\sigma_{3}^{2}\right)}- \\
C_{3} & =\frac{1}{2 \sigma_{3}\left(\sigma_{1}-\sigma_{3}\right)\left(\sigma_{1}{ }^{*}-\sigma_{3}\right)} \\
D_{3} & =\frac{1}{\left(1-\sigma_{1}\right)\left(1-\sigma_{1}{ }^{*}\right)\left(\sigma_{1}-1\right)}=\frac{1}{4 A B} \\
C_{4} & =\frac{-\left(1-\sigma_{3}\right)}{2 \sigma_{3}\left(\sigma_{1}+\sigma_{3}\right)\left(\sigma_{1}{ }^{*}+\sigma_{3}\right)}
\end{aligned}
$$

Complex conjugates are denoted by ()$^{*}$.

Rather than use (3) directly, it is somewhat more informative to examine the behavior for small and large times. Lsing. standard expansions, one finds for $\tau \ll 1$,

$$
\left(m^{\prime} / \bar{m}\right)(\tau) \sim\left[4 / 3(\pi)^{1 / 2}\right](n A B \Delta \beta) \tau^{3 / 2}(\tau \rightarrow 0)
$$

and for large times,

$$
\begin{aligned}
& \frac{m^{\prime}}{\bar{m}}(\tau) \sim n \Delta+4 n A B \Delta\left[\left(D_{1}-C_{1}\right) e^{s_{1} r}+\right. \\
& \left.\left(D_{1}^{*}-C_{1}^{*}\right) e^{\beta^{*} \tau}+\frac{C_{4}}{(\pi \tau)^{1 / 2}} e^{-\tau / 4}-\sigma_{3} C_{3} e^{-\beta \tau}\right](\tau \rightarrow \infty)
\end{aligned}
$$

where

$$
s_{1}=-a / 2+i\left[A^{2} B-a(A+a / 4)\right]^{1 / 2}
$$

and

$$
a=A(1+B)-A^{2}(1-B)^{2}
$$

The middle three terms in (7) are transients characteristic of the propellant; they are all stable only if the real part $-a / 2$ of $s_{1}$ is negative, i.e., $a>0$. The boundary of this kind of "spontaneous" or "intrinsie" instability is defined by $a=0$. The frequency of the transient oscillations, given by the imaginary part of $s_{1}$, is then $\Omega=A(B)^{1 / 2}$ as pointed out in Ret. 1.

The term $n \Delta$ is simply the small change of burning rate due to a change of pressure $p^{\prime} / \bar{p}=\Delta$ when the burning law 


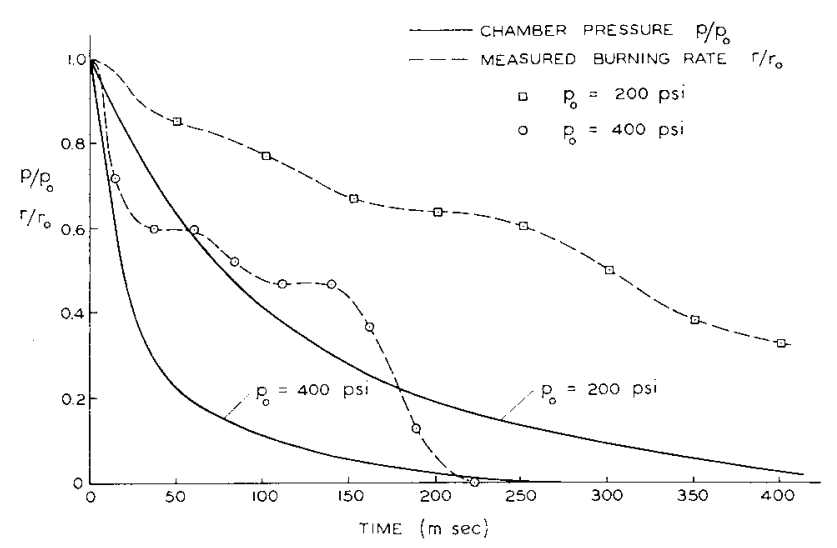

Fig. 1 Measured chamber pressure and instantaneous burning rate.

$m \sim p^{\prime \prime}$ applies. Finally, the last term in $(7)$ is, of course, the "forced" response.

Note that Eq. (6) shows, as one would anticipate, that both the change and rate of change of mass flux (i.e., linear burning rate) vanish at the initiation of the exponential change of in-chamber pressure. Finite changes initially require impulsive changes of pressure. Recent numerical results ${ }^{4-6}$ are defective in this respect, showing finite slopes at $t=0$.

The first two terms in the brackets of Eq. (7) represent an oscillation, which can be written explicitly as

$$
8 n A B \Delta D_{1}-C_{1}^{\prime} e^{-a r_{i} / 2} \cos (\Omega \tau+\phi)
$$

where $\Omega_{t}=A\left[B-a\left(A+a_{i} / 4\right) / A^{2}\right]^{1 / 2}$ and $\tan \phi$ is $\operatorname{Re}\left(D_{1}-\right.$ $C_{1}$ ) divided by $\operatorname{Im}\left(D_{1}-C_{1}\right)$. This transient oscillation, a characteristic of the propellant, also does not appear in most of the numerical results cited; the sole exception is Fig. 14 of Ref. 5 , in which a $1 \%$ step change of pressure produced an oscillation having initially an amplitude only a bit less than $1 \%$. Some suspicion is cast on the remaining nonlinear results until these deficiencies are explained.

However, a more interesting question is whether measurement of the burning rate in response to a pressure perturbation like (2) will rield information about the parameters $A$ and $B$. Clearly, the data must be quite good and impossible to obtain by conventional means. Recently, however, a technique based on the use of microwaves has been developed. ${ }^{7}$ It appears possible to resolve changes of burning rate in times of a few milliseconds. Two results for separate firings in a small chamber are shown in Fig. 1. The propellant sample was a small strand, and the approximately exponential decrease of pressure was achieved by puncturing a diaphragm at an exhaust port.

According to the measurements shown, the instantaneous burning rate exhibits an oscillation that does not appear in the pressure trace. Since the instrumentation had an adequate response time and sensitivity to measure pressure oscillations, one must tentatively conclude that they were negligibly small, if not absent. Hence, the oscillation of burning rate cannot, at least for the present, be associated with an $L^{*}$ instability. Within the linear approximations, therefore, the oscillations must evidently be identified as the transient vibration shown in Eq. (10). The data indicate that the oscillations decay very slowly, implying at $\sim 0$ in Eq. (10). For the propellant used, $\bar{r} \sim 0.18 \mathrm{in} . / \mathrm{sec}$ at $200 \mathrm{psi}$ and $0.24 \mathrm{in.} / \mathrm{sec}$ at $400 \mathrm{psi}$. With $\varkappa \approx 5 \times 10^{-4}$ in. $2 / \mathrm{sec}$, the characteristic time (really for the thermal wave in the solid) is roughly 15 msec. Thus, it is necessary that in fact $a \sim 0$ if $a \tau \sim 0$ for the tests shown. In that case, $\Omega_{t} \approx A(B)^{1 / 2}$ so the real frequency is $\omega \sim \tilde{r}^{2} A(B / \varkappa)^{1 / 2}$ which should increase with pressure if $A$ and $B$ are constant. For the data shown, $\omega \sim 44 \mathrm{rad} / \mathrm{sec}$ at $200 \mathrm{psi}$ and $125 \mathrm{rad} / \mathrm{sec}$ at 400 psi, thus verifying this conclusion.

With the given values, one finds $A(B)^{1 / 2} \sim 0.695$ at 200 psi and 1.07 at 400 psi. This trend of $A(B)^{1 / 2}$ has been found in other experiments," but although $A$ depends on surface temperature, and hence on mean pressure, no existing theory can accommodate such a large change with pressure. Typical values for $A$ and $B$ which have been determined elsewhere ${ }^{2}$ are $A \sim 10-15$ and $B \sim 0.7-0.9$; the value $A(B)^{1 / 2} \sim 1$ is therefore at least reasonable, although perhaps a bit small.

Since $A$ and $B$ are related by $(9)$ as well as by the expression for $\Omega_{t}$, measurement of the frequency and rate of decay of the transient oscillation may provide directly the values of both $A$ and $B$.

Thus, the data examined seem to support qualitatively the idea that the transient behavior of the thermal wave in the solid has been observed during the pressure decay, and that the response of the buming surface is roughly described by the simplest kind of analysis leading to Eq. (1). Whether or not extinction finally occurs cannot be determined by the linear analysis.

If the preceding argument is valid, and if sufficiently accurate data can be obtained, then the experimental technique of Ref. 7 may be superior to both the $L^{*}$ bumer and the $T$ burner for establishing the stability characteristics of a propellant. Measurements of the frequency and the slope of the envelope (i.e., $a$ ) of the oscillation give the values of both $A$ and $B$ for a given pressure level, with only a single firing. Several firings in a $T$ burner are required, and the $L^{*}$ burner is seriously restricted to quite low pressures. Once $A$ and $B$ are found, the entire response function is determined by $\mathrm{Eq}$. (1).

\section{References}

${ }^{1}$ Culick, F. E. C., "A Review of Calculations for Cnsteady" Burning of a Solid Propellant," AtAA Journal, Vol. 6, No. 12, Dec. 1968, pp. 2241-22.5.

${ }^{2}$ Berkstead, M. B. and Culick, F. E. C., "A Comparison of Analysis and Experiment for the Response Function of a Burning Propellant," Rept. NWC TP 4.331, May 1968, U.S. Naval Weapons Center.

"Denison, M. R. and Baum, E., "A simplified Model of Lnstable Burning in Solid Propellants," ARS Journal, Vol. 31, No. 8, Aug. 1961, pp. 1112-1122.

"Krier, H. et al., "Nonsteady Burning Phenomenon of Solid Propellants: Theory and Experiment," AIAA Journal, Vol. 6, No. 2, Feb. 1968, pp. 278-28;.

"Wooldridge, C. E. and Marxman, G. A., "Nonlinear Solid Propellant Burning Rate Behavior During Abrupt Pressiure Excursions," Paper 69-172, 1969, AIAA.

${ }^{6}$ Merkle, C. L., Turk, S. L., and summerfield, M., "Extinguishment of Solid Propellants by Depressurization: Effects of Propellant Parameters," Paper 69-176, 1969, AIAA.

7 shelton, S., presented at 3rd ICRPG/AlAA Joint Propulsion Conference, Atlantic City, N. J., June 1968; also, Strand, L., and Dowler, W., private communication, Jet Propulsion Lab., California Institute of Technology, Pasadena, Calif. 